\title{
Thyroid dysfunction in patients with suspected or documented supraventricular tachyarrhythmia
}

\author{
Hye Bin Gwag ${ }^{1}$, Ji Eun Jun², Youngjun Park³, Seong Soo Lee ${ }^{3}$, Seung-Jung Park³, June Soo Kim³, \\ Kyoung-Min Park ${ }^{3}$ and Young Keun $\mathrm{On}^{3^{*}}$ (i)
}

\begin{abstract}
Background and objectives: The association between thyroid dysfunction and supraventricular tachyarrhythmia (SVT) other than atrial fibrillation (AF) is not well elucidated. We hypothesized that patients with suspected or documented SVT have undetected thyroid dysfunction more frequently than general population.

Subjects and methods: Patients with symptoms suggestive of SVT other than AF who were planned to undergo an EP study at our center were eligible for this study. Patients with known thyroid disease, medications affecting thyroid function test (TFT) or atrial fibrillation were excluded. The primary outcome was prevalence of TFT abnormalities in the study population. The secondary outcome was prevalence of clinically significant arrhythmia during the EP study.

Results: A total of 533 patients were analyzed. Patients were divided into the two groups according to TFT results. Fifty-six (10.5\%) patients had abnormal TFT results (thyroid dysfunction group), while 477 (89.5\%) had normal thyroid function (euthyroidism group). The overall prevalence of thyroid dysfunction was $10.5 \%$. The thyroid dysfunction group showed a tendency for higher induction of clinically significant SVT than the euthyroidism group. However, thyroid dysfunction was not an independent predictor of clinically significant SVT during EP study.

Conclusion: In this study, patients with suspected SVT who were planned for EP study had a relatively high incidence of thyroid dysfunction. Clinically significant arrhythmia tended to be induced more frequently in the thyroid dysfunction group than in the euthyroidism group. Further investigations are needed to verify the clinical implications of TFT in patients with SVT other than AF.
\end{abstract}

Keywords: Thyroid function, Palpitation, Tachyarrhythmia, Electrophysiology

\section{Introduction}

Many people experience palpitations at some point in their lives. Palpitations, however, are not always due to an arrhythmia, and not all arrhythmias result in palpitations. Furthermore, while some patients are diagnosed by electrocardiogram, others only have symptoms of arrhythmias. Electrophysiology (EP) studies help to elucidate the presence or mechanism of arrhythmia in these patients.

\footnotetext{
*Correspondence: yk.on@samsung.com

${ }^{3}$ Division of Cardiology, Department of Medicine, Samsung Medical Center, Sungkyunkwan University School of Medicine, \#81 Irwon-ro Gangnam-gu, Seoul 06351, Republic of Korea

Full list of author information is available at the end of the article
}

Thyroid dysfunction, particularly hyperthyroidism, can cause palpitations, which are one of the common symptoms of thyroid disease [1]. There is a well-established relationship between atrial fibrillation (AF) and thyroid dysfunction. Therefore, a recent guideline recommends the evaluation of thyroid function in AF patients [2-4]. Although supraventricular tachyarrhythmias (SVT) other than AF are a common cause of palpitations, the association of non-AF SVTs and thyroid dysfunction has not been well elucidated. The current guidelines recommend ruling out hyperthyroidism in cases of inappropriate sinus tachycardia or premature extra beats $[5,6]$. There are a limited number of studies and case reports that investigate the relationship between thyroid dysfunction

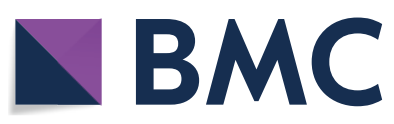

(c) The Author(s) 2019. This article is distributed under the terms of the Creative Commons Attribution 4.0 International License (http://creativecommons.org/licenses/by/4.0/), which permits unrestricted use, distribution, and reproduction in any medium, provided you give appropriate credit to the original author(s) and the source, provide a link to the Creative Commons license, and indicate if changes were made. 
and SVT other than AF [7-10]. At our institution, thyroid function test (TFT) has been routinely performed in all patients undergoing EP studies in the work-up of suspected or documented SVT. In this study, we investigated thyroid function abnormality in patients with non-AF SVT to evaluate the clinical value of thyroid function test in this setting.

\section{Materials and methods}

Patients with symptoms suggestive of SVT other than AF who were scheduled to undergo an EP study at our institution between January 2014 and May 2017 were eligible for this study. Patients who met any of the following criteria were excluded from the analysis: (1) previous history of thyroidectomy or known thyroid dysfunction, (2) medications that influence TFT, including thyroid hormone, antithyroid drugs, amiodarone, or steroid, (3) no TFT results within 2 months of the EP study, or (4) previous history of AF.

TFT was performed unless patients had undergone TFT within the prior 2 months at our institution. Serumfree T4, total T3, and thyroid stimulating hormone (TSH) were measured using chemiluminescent immunoassay (ADVIA Centaur XP, Siemens). Based on the reference intervals from a recent large nationwide epidemiological study, the normal TFT ranges were defined as 0.62$6.86 \mathrm{mU} / \mathrm{L}$ for TSH level and $0.96-1.60 \mathrm{ng} / \mathrm{dL}$ for FT4 level [11]. Euthyroidism was defined as TSH and FT4 levels in the normal range. Subclinical hypothyroidism was defined as TSH $>6.86 \mathrm{mU} / \mathrm{L}$ with normal FT4 level, and subclinical hyperthyroidism as TSH $<0.62 \mathrm{mU} / \mathrm{L}$ with normal FT4 level.

All EP studies were performed after discontinuation of antiarrhythmic drugs. One duodecapolar catheter (or two decapolar catheters) was placed in the high right atrium and coronary sinus. Two quadripolar electrode catheters were placed in the right ventricular apex and $\mathrm{His}$ area, respectively. Isoproterenol was infused at a rate of $2-5 \mathrm{mcg} / \mathrm{min}$, unless a significant tachyarrhythmia could be induced by programmed stimulation. If possible, radiofrequency ablation was performed for spontaneous or induced tachyarrhythmia.

A trained study coordinator collected the clinical and laboratory data using a standardized case report form and protocol. The baseline electrocardiograms were reviewed by two EP specialists. The primary outcome was prevalence of TFT abnormality in the study population. The secondary outcome was prevalence of clinically significant arrhythmia during EP study. Two EP specialists determined whether arrhythmias were clinically significant based upon consideration of the following characteristics: (1) sustained arrhythmia during EP study, (2) reproducible induction by programmed stimulation, (3) arrhythmia generating palpitation similar to previous symptoms, or (4) compatible with the previously documented electrocardiogram. The local institutional review board approved the study protocol and waived the requirement for informed consent (IRB No. 2017-10-146).

Continuous variables are presented as median with interquartile range or mean \pm standard deviation, whereas categorical variables are presented as number and percentage. Continuous variables were compared between groups using Student's $t$-test. Categorical data were compared between groups using Fisher's exact test or the Chi-square test, as appropriate. A logistic regression model was used to determine the independent predictors of clinically significant arrhythmia during EP study. Variables with a $p$ value $<0.1$ on univariate analysis were included in the multivariate analysis. All tests were two-sided, and $p$ values $<0.05$ were considered statistically significant. IBM SPSS Statistics software version 23 (IBM Corporation, Armonk, NY, USA) was used for statistical analysis.

\section{Results}

Baseline characteristics of the study population

A total of 639 patients with suspected or documented SVT underwent EP study. We excluded patients with history of thyroidectomy or known thyroid disease $(n=33)$, patients taking medications that affect the TFT $(n=9)$, patients without TFT results $(n=33)$, and patients with a previous history of AF $(n=31)$. Of the final 533 patients, 56 (10.5\%) had abnormal TFT results (thyroid dysfunction group), while 477 (89.5\%) had normal TFTs (euthyroidism group) (Fig. 1). The types of thyroid dysfunction are shown in Fig. 2. The most common type of thyroid dysfunction was subclinical hyperthyroidism (80.4\%). The mean duration between TFT and EP study was $1.3 \pm 2.0$ days in the thyroid dysfunction group and $1.4 \pm 3.2$ days in the euthyroidism group $(p=0.97)$. There was no significant difference in the baseline clinical characteristics between the two TFT groups (Table 1).

\section{EP study results}

EP study results are shown in Table 2. Paroxysmal supraventricular tachycardia was the most common SVT, while atrial fibrillation or flutter was the second most commonly induced SVT in both groups. The thyroid dysfunction group showed a tendency for higher induction of clinically significant SVT than the euthyroidism group $(94.6 \%$ vs. $86.6 \%, p=0.09)$. When patients with previously documented SVT were analyzed separately, clinically significant SVT could be induced in all patients in the thyroid dysfunction group. In contrast, we were 

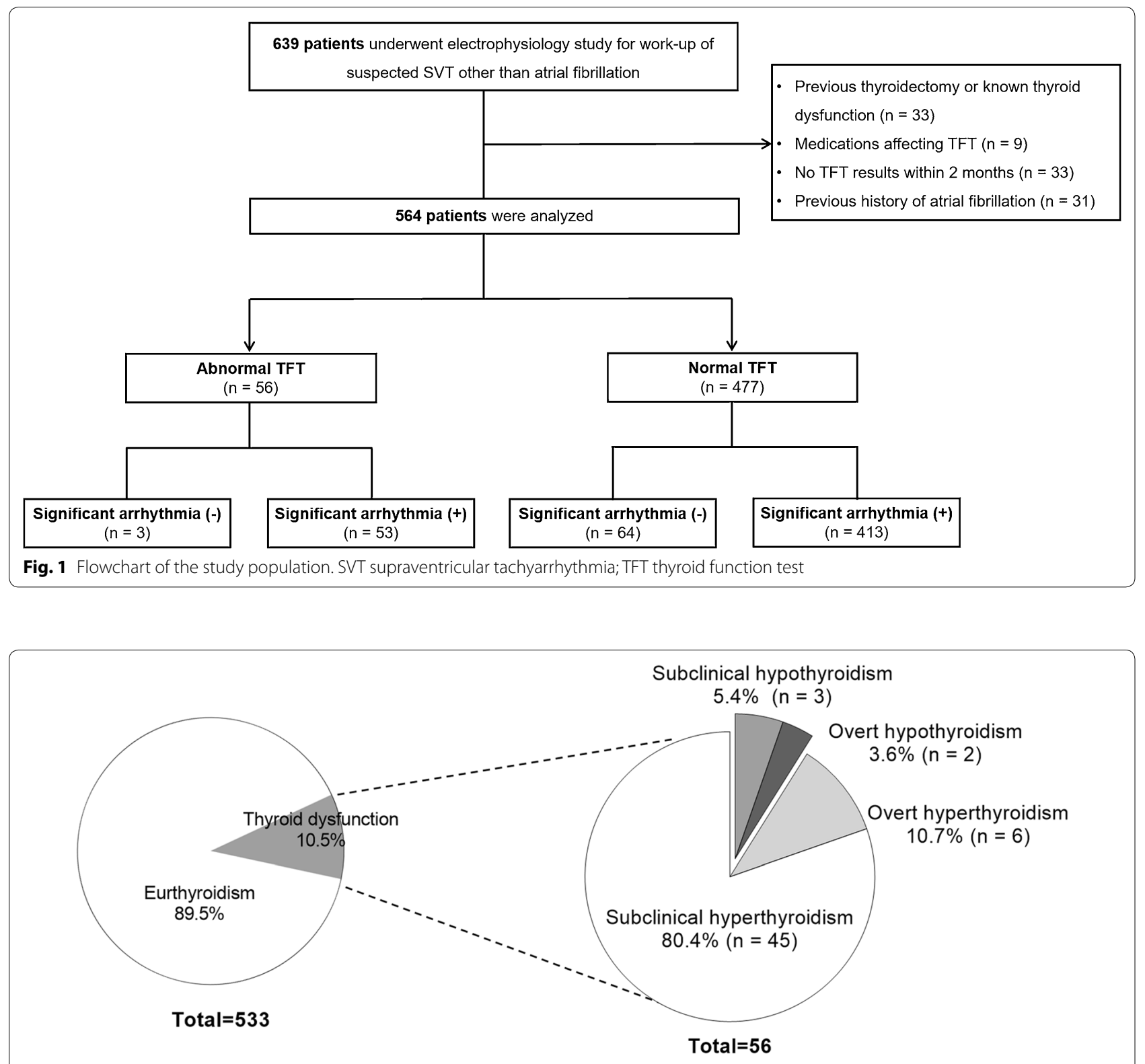

Fig. 2 Types of thyroid dysfunction

unable to induce a significant SVT in $31(9.2 \%)$ patients in the euthyroidism group.

On multivariate logistic regression analysis, a previous documentation of SVT was significantly associated with induction of clinically significant SVT (Table 3).

\section{Discussion}

In the present study, we investigated thyroid function abnormality in patients with suspected or documented SVT undergoing EP study. The major findings of this study were: (1) The prevalence of newly detected thyroid dysfunction in the study population was $10.5 \%$, (2) clinically significant arrhythmia tended to be induced more frequently in the thyroid dysfunction group than the euthyroidism group, while (3) thyroid dysfunction was not an independent predictor of clinically significant arrhythmia during EP study.

Since the relationship between AF and thyroid dysfunction is well established, TFT is recommended in all AF patients [2-4]. Recent studies suggest that the risk of $\mathrm{AF}$ is not only increased in overt hyperthyroidism, but also in subclinical hyperthyroidism or high normal euthyroid states $[3,12,13]$. Elevated thyroid hormone is believed to play a role in AF through several mechanisms, 
Table 1 Baseline characteristics according to thyroid function

\begin{tabular}{|c|c|c|c|}
\hline & $\begin{array}{l}\text { Euthyroidism } \\
(n=477)\end{array}$ & $\begin{array}{l}\text { Thyroid dysfunction } \\
(n=56)\end{array}$ & $p$ Value \\
\hline Sex (male) & $215(45.1)$ & $19(33.9)$ & 0.12 \\
\hline Age (years) & $45.9 \pm 15.4$ & $44.1 \pm 16.6$ & 0.41 \\
\hline Body mass index (kg/m2) & $23.8 \pm 3.5$ & $23.1 \pm 3.0$ & 0.20 \\
\hline Hypertension & $75(15.7)$ & $9(16.1)$ & $>0.999$ \\
\hline Diabetes & $26(5.5)$ & $1(1.8)$ & 0.34 \\
\hline \multicolumn{4}{|l|}{ Echocardiographic results $^{\dagger}$} \\
\hline Presence of cardiomyopathy & $5(1.2)$ & $0(0)$ & $>0.999$ \\
\hline LVEF (\%) & $63.5 \pm 6.5$ & $65.1 \pm 4.9$ & 0.09 \\
\hline LAVI (ml/m2) & $28.0 \pm 8.8$ & $28.8 \pm 8.4$ & 0.52 \\
\hline Documented SVT & $336(70.4)$ & $40(71.4)$ & $>0.999$ \\
\hline Pre-excitation of QRS & $56(11.7)$ & $6(10.7)$ & 0.84 \\
\hline Previous ablation of SVT & $26(5.5)$ & $1(1.8)$ & 0.34 \\
\hline \multicolumn{4}{|l|}{ Current medications } \\
\hline Beta blocker & $46(9.6)$ & $8(14.3)$ & 0.35 \\
\hline Calcium channel blocker & $178(37.3)$ & $25(44.6)$ & 0.31 \\
\hline Other antiarrhythmic drugs & $20(4.2)$ & $2(3.6)$ & $>0.999$ \\
\hline
\end{tabular}

Values are reported as mean \pm SD or $\mathrm{n}(\%)$

LAVI Left atrial volume index; LVEF left ventricular ejection fraction; SVT supraventricular tachyarrhythmia

${ }^{+}$Echocardiographic results were not available in 65 patients ( $12.2 \%$ of total patients)

including an increase in sympathetic tone, ectopic atrial beats, alteration of ionic channels, or a decreased atrial refractory period $[14,15]$. However, the current guidelines for management of SVT do not recommend routine evaluation of thyroid function except in the case of inappropriate sinus tachycardia or premature extra beats $[5$, 6]. This result might be attributable to a lack of relevant data from only a small number of retrospective studies and case reports [7-10]. Regardless, in our institution, TFT is routinely performed in patients undergoing EP study for suspected or documented SVT. This practice is based on the assumption that thyroid dysfunction may also affect the prevalence of SVT by similar mechanisms that increases the risk of AF. Ectopic beats resulting in conduction delays can initiate re-entrant tachycardia. Increased autonomic tone can also influence the occurrence of tachyarrhythmia associated with enhanced automaticity. Furthermore, in patients with suspected, but not documented SVT, it is difficult to distinguish paroxysmal AF by symptoms alone. More than 600 patients with suspected SVT underwent TFT according to the protocol at our institution. We subsequently analyzed these patients to verify the clinical utility of this routine practice.

The overall prevalence of TFT abnormality in this study was $10.5 \%$. The prevalence of each type of thyroid dysfunction was relatively higher in this study than that of the general Korean population in similar age-groups [11]. In particular, subclinical hyperthyroidism was far more prevalent in our study population than it was in the general population $(8.4 \%$ vs. $2.9 \%)$. This is a simple comparison of numerical values between two different populations. Nevertheless, we believe that it warrants additional analysis, including that with a matching control group. In this study, the thyroid dysfunction group had a tendency for higher induction rate of clinically significant SVT than did the euthyroidism group, irrespective of previous documentation of SVT. Several previous reports have implied that there is an association between thyroid dysfunction and non-AF SVT. Furthermore, some reports have suggested that antithyroid medications or thyroid replacement therapy could alleviate paroxysms of such SVTs $[8,10,16,17]$. One study even reported that radioactive iodine could be an effective agent to prevent attack of recurrent paroxysmal supraventricular tachycardia in euthyroid patients [17]. However, we are unable to fully explain how thyroid dysfunction affects non-AF SVTs. There are no previous studies that have addressed this mechanism clearly. We suggest it is possible that thyroid dysfunction increases the prevalence of SVT by increasing the frequency of premature beats and altering the autonomic tone, both of which also contribute to the risk of AF. Subclinical hyperthyroidism comprised the majority of thyroid dysfunction in our study (Fig. 2). Therefore, further investigation is needed to determine whether subclinical dysfunction affects arrhythmogenesis. One study suggests that, 
Table 2 Electrophysiology study results according to thyroid function

\begin{tabular}{|c|c|c|c|}
\hline & Euthyroidism & $\begin{array}{l}\text { Thyroid } \\
\text { dysfunction }\end{array}$ & $p$ Value \\
\hline \multicolumn{4}{|l|}{ Total population } \\
\hline Patient number & 477 & 56 & \\
\hline Significant SVT & $413(86.6)$ & $53(94.6)$ & 0.09 \\
\hline \multicolumn{4}{|l|}{ Any $S V T^{+}$} \\
\hline PSVT & $375(78.6)$ & $47(83.9)$ & 0.39 \\
\hline Atrial fibrillation or flutter & $48(10.1)$ & $8(14.3)$ & 0.36 \\
\hline Atrial tachycardia & $38(8.0)$ & $4(7.1)$ & $>0.999$ \\
\hline Junctional tachycardia & $7(1.5)$ & $0(0)$ & $>0.999$ \\
\hline $\begin{array}{l}\text { Inappropriate sinus tachy- } \\
\text { cardia }\end{array}$ & $1(0.2)$ & $1(1.8)$ & 0.20 \\
\hline \multicolumn{4}{|c|}{ Patients with previously documented SVT } \\
\hline Patient number & 336 & 40 & \\
\hline Significant SVT & $305(90.8)$ & $40(100)$ & 0.06 \\
\hline \multicolumn{4}{|l|}{ Any SVT $T^{\ddagger}$} \\
\hline PSVT & $278(82.7)$ & $35(87.5)$ & 0.51 \\
\hline Atrial fibrillation or flutter & $36(10.7)$ & $6(15.0)$ & 0.43 \\
\hline Atrial tachycardia & $28(8.3)$ & $4(10.0)$ & 0.76 \\
\hline Junctional tachycardia & $6(1.8)$ & $0(0)$ & $>0.999$ \\
\hline $\begin{array}{l}\text { Inappropriate sinus tachy- } \\
\text { cardia }\end{array}$ & $0(0)$ & $0(0)$ & N/A \\
\hline
\end{tabular}

Values are reported as $\mathrm{n}(\%)$

N/A Not applicable; PSVT paroxysmal supraventricular tachycardia; SVT supraventricular tachyarrhythmia

${ }^{+}$Eight cases of atrial fibrillation or flutter, 4 of atrial tachycardia, and 5 of junctional tachycardia were regarded as not clinically significant

${ }^{\ddagger}$ Four cases of atrial fibrillation or flutter, 4 of atrial tachycardia, and 4 of junctional tachycardia were regarded as not clinically significant

in euthyroid patients, unusual irritability or sensitivity of the cardiac muscle can result in arrhythmogenesis. Another study found that endogenous subclinical hyperthyroidism can affect intrinsic cardiac morphology and function [17]. However, these hypotheses are unsubstantiated and require further investigation.

In multivariate logistic regression analysis, thyroid dysfunction was not a significant predictor of clinically significant arrhythmia during EP study $(p=0.10)$. In contrast, documented SVT was significantly associated with clinically significant arrhythmia. We believe that unmeasured variables could have affected the result. For example, patients with overt thyroid dysfunction tend to be symptomatic with weight change, goiter, heat/cold intolerance, or bowel habit change. These patients are more likely to be treated with antithyroid medications or thyroid replacement therapy than they are to be evaluated with an EP study. This reason likely explains why we found subclinical thyroid disease more often than overt thyroid dysfunction in this study. We could not acquire data regarding the detailed reasons for EP study, characteristics, frequency, duration, or severity of the suspected SVT episodes. These data would be meaningful with regard to the homogeneity of the study population. For example, while some patients might have been referred for EP study after only a single episode of palpitations, others may have had recurrent episodes. We may have obtained more significant results if we had performed a subgroup analysis using those data.

To the best of our knowledge, this is the largest study to address the association between thyroid dysfunction and the prevalence of non-AF SVT. We suggest that TFT needs to be considered in patients with suspected or documented supraventricular tachyarrhythmia considering the tendency of higher induction of clinically significant arrhythmia in those with thyroid dysfunction than those without. Although this study cannot provide conclusive evidence regarding whether thyroid dysfunction is associated with SVTs other than AF, we believe that it indicates the need for further discussion about the clinical significance of TFT in patients with suspected SVT.

This study has several limitations. First, this was a cross-sectional single-center study; therefore, the causal relationship between thyroid dysfunction and SVT could not be definitively proven. We were unable to investigate the follow-up outcomes after EP study, and follow-up TFT data were unavailable. Our results are also possibly subject to selection bias, because the study population

Table 3 Predictors of clinically significant arrhythmia during electrophysiology study

\begin{tabular}{|c|c|c|c|c|}
\hline & Odds ratio $(95 \% \mathrm{Cl})$ & $p$ Value & Odds ratio $(95 \% \mathrm{Cl})$ & $p$ Value \\
\hline Thyroid dysfunction & $2.74(0.83-9.02)$ & 0.10 & $2.79(0.84-9.30)$ & 0.10 \\
\hline Male & $1.10(0.66-1.85)$ & 0.71 & & \\
\hline Previous ablation of SVT & $0.48(0.19-1.24)$ & 0.13 & & \\
\hline Documented SVT & $3.31(1.96-5.59)$ & $<0.001$ & $3.33(1.97-5.62)$ & $<0.001$ \\
\hline Pre-excitation of QRS & $0.97(0.44-2.13)$ & 0.93 & & \\
\hline Cardiomyopathy & $0.58(0.06-5.32)$ & 0.63 & & \\
\hline LVEF & $1.02(0.98-1.06)$ & 0.44 & & \\
\hline
\end{tabular}

CI Confidence interval; LVEF left ventricular ejection fraction; SVT supraventricular tachyarrhythmia 
consisted of patients admitted to a tertiary university hospital for EP study. Patients with symptoms of overt thyroid dysfunction may have been excluded at the outpatient clinic. Similarly, patients with tolerable symptoms could have been medically followed without an EP study. Therefore, our results are not necessarily generalizable to all patients with suspected or documented SVT. Second, the TFT reference ranges used in this study might not apply to other ethnic groups because of differences attributable to genetics and iodine intake. Lastly, an inability to induce SVT during EP study does not equate to an absence of SVT, but rather low arrhythmia inducibility. An SVT could not be induced during the EP studies of 31 patients with previously documented SVT (9.2\%).

\section{Abbreviations}

AF: atrial fibrillation; EP: electrophysiology; SVT: supraventricular tachyarrhythmia; TFT: thyroid function test; TSH: thyroid stimulating hormone.

\section{Acknowledgements}

None.

\section{Authors' contributions}

On YK and Kim JS contributed to conceptualization. Gwag HB and Park SJ contributed to data curation and formal analysis. Gwag HB, Park Y, and Lee SS contributed to investigation. On YK and Kim JS contributed to supervision. Gwag HB and Park SJ contributed to validation and visualization. Gwag HB contributed to writing —original draft. On YK and Park KM contributed to writing-review and editing. All authors read and approved the final manuscript.

\section{Funding}

None.

\section{Availability of data and materials}

All data generated or analyzed during this study are included in this published article [and its supplementary information files.

\section{Ethics approval and consent to participate}

The Institutional Review Board at Samsung Medical Center approved the study protocol (IRB No. 2017-10-146). Informed consent was waived by the board.

\section{Consent for publication}

Not applicable.

\section{Competing interests}

The authors declare that they have no competing interests.

\begin{abstract}
Author details
${ }^{1}$ Division of Cardiology, Department of Internal Medicine, Samsung Changwon Hospital, Sungkyunkwan University School of Medicine, Changwon, Republic of Korea. ${ }^{2}$ Department of Endocrinology and Metabolism, Kyung Hee University Hospital, Kyung Hee University School of Medicine, Seoul, Republic of Korea. ${ }^{3}$ Division of Cardiology, Department of Medicine, Samsung Medical Center, Sungkyunkwan University School of Medicine, \#81 Irwon-ro Gangnam-gu, Seoul 06351, Republic of Korea.
\end{abstract}

Received: 1 Auqust 2019 Accepted: 25 November 2019 Published online: 10 December 2019

\section{References}

1. Mai VQ, Burch HB. A stepwise approach to the evaluation and treatment of subclinical hyperthyroidism. Endocr Pract. 2012;18:772-80.
2. Kim EJ, Lyass A, Wang N, Massaro JM, Fox CS, Benjamin EJ, Magnani JW. Relation of hypothyroidism and incident atrial fibrillation (from the Framingham Heart Study). Am Heart J. 2014;167:123-6.

3. Selmer C, Olesen JB, Hansen ML, Lindhardsen J, Olsen AM, Madsen JC, Faber J, Hansen PR, Pedersen OD, Torp-Pedersen C, Gislason GH. The spectrum of thyroid disease and risk of new onset atrial fibrillation: a large population cohort study. BMJ. 2012;345:e7895.

4. Kirchhof P, Benussi S, Kotecha D, Ahlsson A, Atar D, Casadei B, Castella M, Diener HC, Heidbuchel H, Hendriks J, Hindricks G, Manolis AS, Oldgren J, Popescu BA, Schotten U, Van Putte B, Vardas P, Agewall S, Camm J, Baron Esquivias G, Budts W, Carerj S, Casselman F, Coca A, De Caterina R, Deftereos S, Dobrev D, Ferro JM, Filippatos G, Fitzsimons D, Gorenek B, Guenoun M, Hohnloser SH, Kolh P, Lip GY, Manolis A, McMurray J, Ponikowski P, Rosenhek R, Ruschitzka F, Savelieva I, Sharma S, Suwalski P, Tamargo JL, Taylor CJ, Van Gelder IC, Voors AA, Windecker S, Zamorano JL, Zeppenfeld K. 2016 ESC Guidelines for the management of atrial fibrillation developed in collaboration with EACTS. Europace. 2016;18:1609-78.

5. Blomstrom-Lundqvist C, Scheinman MM, Aliot EM, Alpert JS, Calkins H, Camm AJ, Campbell WB, Haines DE, Kuck KH, Lerman BB, Miller DD, Shaeffer CW, Stevenson WG, Tomaselli GF, Antman EM, Smith SC Jr, Alpert JS, Faxon DP, Fuster V, Gibbons RJ, Gregoratos G, Hiratzka LF, Hunt SA, Jacobs AK, Russell RO Jr, Priori SG, Blanc JJ, Budaj A, Burgos EF, Cowie M, Deckers JW, Garcia MA, Klein WW, Lekakis J, Lindahl B, Mazzotta G, Morais JC, Oto A, Smiseth O, Trappe HJ, European Society of Cardiology Committee N-HRS. ACC/AHA/ESC guidelines for the management of patients with supraventricular arrhythmias-executive summary. A report of the American college of cardiology/American heart association task force on practice guidelines and the European society of cardiology committee for practice guidelines (writing committee to develop guidelines for the management of patients with supraventricular arrhythmias) developed in collaboration with NASPE-Heart Rhythm Society. J Am Coll Cardiol. 2003;42:1493-531.

6. Page RL, Joglar JA, Caldwell MA, Calkins H, Conti JB, Deal BJ, Estes NAM 3rd, Field ME, Goldberger ZD, Hammill SC, Indik JH, Lindsay BD, Olshansky B, Russo AM, Shen WK, Tracy CM, Al-Khatib SM. 2015 ACC/AHA/HRS guideline for the management of adult patients with supraventricular tachycardia: executive summary: a report of the American College of Cardiology/American Heart Association Task Force on Clinical Practice Guidelines and the Heart Rhythm Society. J Am Coll Cardiol. 2016;67:1575-623.

7. Benvenga S, Granata A, Trimarchi F. Long-standing recurrent paroxysmal supraventricular tachycardia disappearing after cystic degeneration of an episodically hypersecreting toxic adenoma of the thyroid. G Ital Cardiol. 1984;14:337-40.

8. Vanin LN, Smetnev AS, Sokolov SF, Kotova GA. Masenko VP [Study of thyroid function in patients with paroxysmal supraventricular tachycardia]. Kardiologiia. 1989;29:71-4.

9. Ramkumarsingh Tomar L, Thondikulam Gopalakrishnan S, Aggarwal A. Paroxysmal Supraventricular tachycardia in Hypothyroidism: a Case Report. J Cardiovasc Dis Res. 2015;6:156-8.

10. von Olshausen K, Bischoff S, Kahaly G, Mohr-Kahaly S, Erbel R, Beyer J, Meyer J. Cardiac arrhythmias and heart rate in hyperthyroidism. Am J Cardiol. 1989:63:930-3.

11. Kim WG, Kim WB, Woo G, Kim H, Cho Y, Kim TY, Kim SW, Shin MH, Park JW, Park HL, Oh K, Chung JH. Thyroid stimulating hormone reference range and prevalence of thyroid dysfunction in the korean population: Korea National Health and Nutrition Examination Survey 2013 to 2015. Endocrinol Metab (Seoul). 2017;32:106-14.

12. Collet TH, Gussekloo J, Bauer DC, den Elzen WP, Cappola AR, Balmer P, lervasi G, Asvold BO, Sgarbi JA, Volzke H, Gencer B, Maciel RM, Molinaro S, Bremner A, Luben RN, Maisonneuve P, Cornuz J, Newman AB, Khaw KT, Westendorp RG, Franklyn JA, Vittinghoff E, Walsh JP, Rodondi N, Thyroid Studies C. Subclinical hyperthyroidism and the risk of coronary heart disease and mortality. Arch Intern Med. 2012;172:799-809.

13. Asvold BO, Vatten LJ, Midthjell K, Bjoro T. Serum TSH within the reference range as a predictor of future hypothyroidism and hyperthyroidism: 11-year follow-up of the HUNT Study in Norway. J Clin Endocrinol Metab. 2012:97:93-9.

14. Chen YC, Chen SA, Chen YJ, Chang MS, Chan P, Lin Cl. Effects of thyroid hormone on the arrhythmogenic activity of pulmonary vein cardiomyocytes. J Am Coll Cardiol. 2002;39:366-72. 
15. Reddy V, Taha W, Kundumadam S, Khan M. Atrial fibrillation and hyperthyroidism: a literature review. Indian Heart J. 2017;69:545-50.

16. Osman F, Gammage MD, Sheppard MC, Franklyn JA. Clinical review 142: cardiac dysrhythmias and thyroid dysfunction: the hidden menace? J Clin Endocrinol Metab. 2002;87:963-7.

17. Corday E, Gold H, Jaffe HL. Radioiodine treatment of paroxysmal supraventricular tachycardia in the euthroid patient. Circulation. 1958;17:900-6.

\section{Publisher's Note}

Springer Nature remains neutral with regard to jurisdictional claims in published maps and institutional affiliations.
Ready to submit your research? Choose BMC and benefit from:

- fast, convenient online submission

- thorough peer review by experienced researchers in your field

- rapid publication on acceptance

- support for research data, including large and complex data types

- gold Open Access which fosters wider collaboration and increased citations

- maximum visibility for your research: over $100 \mathrm{M}$ website views per year

At BMC, research is always in progress.

Learn more biomedcentral.com/submissions 